\title{
Identification of a mutation in the WISP3 gene in three unrelated families with progressive pseudorheumatoid dysplasia
}

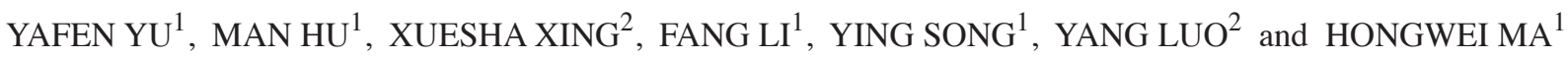 \\ ${ }^{1}$ Department of Developmental Pediatrics, Shengjing Hospital Affiliated to China Medical University, Shenyang, Liaoning 110004; \\ ${ }^{2}$ Department of Medical Genomics, China Medical University, Shenyang, Liaoning 110001, P.R. China
}

Received May 11, 2014; Accepted January 15, 2015

DOI: $10.3892 / \mathrm{mmr} .2015 .3430$

\begin{abstract}
Progressive pseudorheumatoid dysplasia (PPD) is a rare autosomal recessive genetic disease, which is caused by the functional loss or abnormality of Wntl-inducible signaling pathway protein 3 [WISP3 protein (also termed CCN6, OMIM \#603400)]. WISP3 is a member of the cysteine-rich 61/connective tissue growth factor/nephroblastoma overexpressed protein family. Mutations in WISP3 may result in continuous degeneration and loss of articular cartilage. The present study collected clinical data from three patients with PPD from three unrelated families, and WISP3 mutations were detected by polymerase chain reaction and direct sequencing. Overall, five mutations were identified, which consisted of two missense mutations, two nonsense mutations and one duplication mutation, which spanned exons 2, 4 and 5 of WISP3. In family 1 , a compound heterozygosity mutation of WISP3 was detected, and the proband was shown to carry a novel missense mutation: c.667T $>$ G (p.Cys223Gly) and a
\end{abstract}

Correspondence to: Mrs. Hongwei Ma, Department of Developmental Pediatrics, Shengjing Hospital Affiliated to China Medical University, 36 San Hao, Heping Ward, Shenyang, Liaoning 110004, P.R. China

E-mail: mahongwei1960@163.com

Mrs. Yang Luo, Department of Medical Genomics, China Medical University, 92 North $2^{\text {nd }}$ Road, Shenyang, Liaoning 110001, P.R. China

E-mail: luoyang@mail.cmu.edu.cn

Abbreviations: PPD, progressive pseudorheumatoid dysplasia; WISP3, Wntl-inducible signaling pathway protein 3; $\mathrm{CCN}$, cysteine-rich 61/connective tissue growth factor/nephroblastoma overexpressed; PCR, polymerase chain reaction; SEDT, spondyloepiphyseal dysplasia tarda; JIA, juvenile idiopathic arthritis; IGFBP, insulin-like growth factor binding protein domain; VWC, Von Willebrand type C repeat; TSP-1, thrombospondin type I domain; CT, cysteine knot carboxyl terminal; BMP, bone morphogenetic protein; ECM, extracellular matrix; SDS, height standard deviation score

Key words: progressive pseudorheumatoid dysplasia, clinical manifestation, WISP3, mutation, cysteine-rich 61/connective tissue growth factor/nephroblastoma overexpressed, chondrocyte, genetics nonsense mutation: c.857C $>\mathrm{G}$ (p.Ser286*). The other three mutations: c.342T>G (p.Cys114Trp), c.136C >T (p.Gln46*) and c.866dupA (p.Ser290Glufs*13) had previously been identified. Overall, the three patients had similar clinical phenotypes, and no specific correlation between genotype and phenotype was detected. The results of the present study expand the WISP3 mutation spectrum that is associated with PPD and aid in further elucidating the function of WISP3.

\section{Introduction}

Progressive pseudorheumatoid dysplasia (PPD; OMIM \#208230) is also termed spondyloepiphyseal dysplasia tarda with progressive arthropathy, or arthropathy progressive pseudorheumatoid of childhood. PPD is a rare autosomal recessive hereditary disease, which is caused by mutations in the Wntl-inducible signaling pathway protein 3 (WISP3) gene $(1,2)$. The WISP3 gene is located on chromosome $6 \mathrm{q} 22$ and is composed of five coding exons, which encode a 354-amino acid protein. WISP3 is maintains the stability of cartilage by regulating the synthesis of type II collagen and aggrecan, two major matrix components of articular cartilage, in chondrocytes. The incidence rate of PPD in the UK is $1 / 1,000,000$ (3). The disease is asymptomatic at birth; however, progressive enlargement of numerous joints, deformity and limitation of activity emerge with age, without specificity in laboratory examination (4). PPD is often clinically misdiagnosed, and should be distinguished from spondyloepiphyseal dysplasia tarda and juvenile idiopathic arthritis in the early stages of the disease. The aim of the present study was to report on the main clinical and radiographic features of patients referred to our center, and explore the genetic mutation spectrum of WISP3 and the association between phenotype and genotype of PPD in Chinese patients with PPD. The clinical data of three patients with PPD was analyzed, in order to summarize the associated clinical characteristics, and the WISP3 genotype of the patients with PPD was detected.

\section{Patients and methods}

Patients. The present study was approved by the Medical Ethics Committee of Shengjing Hospital Affiliated to China Medical University (Shenyang, China). The three patients with PPD from three different families were referred from the 
Department of Developing Pediatrics of Shengjing Hospital for genetic testing. PPD was determined in the three patients by examining their medical history, laboratory examinations and detection of radiological abnormalities. The laboratory evaluation included routine blood, liver and renal function tests, determination of serum calcium phosphorous and alkaline phosphatase levels, as well as measurements of C-reactive protein, rheumatoid factors, antistreptolysin $\mathrm{O}$ and antinuclear antibodies, in order to exclude connective tissue disease. Radiological investigations (X-rays of the spine, hip, knee and hand) were performed, in order to make a radiological diagnosis of PPD. The clinical and imaging characteristics of each proband were reviewed by two clinical geneticists and two radiologists, respectively. The medical histories and examination results were systematically recorded, and the skeletal $\mathrm{X}$-ray films were reviewed. The patients were screened for WISP3 gene mutations only after informed consent had been obtained from the family members of the patients. All of the available family members were then subjected to restriction analysis. A total of 60 unrelated Han Chinese participants (40 males, 20 females; 5.2-22 years old) who were outpatients at the Shengjing Hospital affiliated to China Medical University over a period of two years, were used as normal controls. Written informed consent was also obtained from the parents of the patients with PPD and the 60 controls prior to blood sampling and DNA analysis. Altogether, 67 DNA samples, including 3 affected individuals, 4 unaffected individuals from two families, and 60 healthy donors were analyzed. DNA was extracted from peripheral white blood cells with conventional methods. The entire coding region (five exons with several alternatively spliced exons) of the WISP3 gene (GeneBank accession number: NC_000006) were sequenced in order to screen mutations using 6 pairs of primers.

Mutation analysis. A total of $2 \mathrm{ml}$ peripheral venous blood was collected from the pediatric patients. Genomic DNA was extracted from the peripheral blood of the pediatric patients, their family members, and the normal controls using the Universal Genomic DNA Extraction kit Ver. 3.0 (Takara Biotechnology Co., Ltd., Dalian, China). Five exons of WISP3 and the exon-intron borders were amplified by polymerase chain reaction (PCR) (TP600; Takara Biotechnology Co., Ltd.). The total volume used for the PCR reaction was $50 \mu 1$, including $25 \mu \mathrm{l} 2 \mathrm{X} \mathrm{GC}$ buffer I, $8 \mu 1 \mathrm{dNTP}(2.5 \mathrm{mmol} / \mathrm{l}), 2.5 \mu \mathrm{l}$ upstream and downstream primers respectively (10 pmol/l), and $0.5 \mu 1 \mathrm{LA}$ Taq ( $5 \mathrm{U} / \mu \mathrm{l}$; Takara Biotechnology Co., Ltd.) The primers were synthesized by Sangon Biological Engineering Technology \& Services Co., Ltd. (Shanghai, China), the primer sequences are shown in Table I. Approximately 40 ng genomic DNA was diluted to $50 \mu \mathrm{l}$ with sterile water. The PCR reaction conditions were set as follows: Predegeneration at $95^{\circ} \mathrm{C}$ for $5 \mathrm{~min}$, followed by degeneration at $94^{\circ} \mathrm{C}$ for $30 \mathrm{sec}$. After annealing for $30 \mathrm{sec}$ at $58^{\circ} \mathrm{C}$, extension was performed at $72^{\circ} \mathrm{C}$ for $1 \mathrm{~min}$. After 35 cycles, a final extension was performed at $72^{\circ} \mathrm{C}$ for $10 \mathrm{~min}$. The PCR products were then sent to Shanghai Sangon Biological Engineering Technology \& Services Co., Ltd. for further analysis.

Shanghai Sangon Biological Engineering Technology \& Services Co., Ltd. (http://genome-test.cse.ucsc.edu/cgi-bin/ hgBlat?command=start) sequenced the PCR products, following detection by $1 \%$ agarose gel electrophoresis. The sequencing results were compared against genomic sequences contained in the UCSC database (http://genome.ucsc.edu/), in order to identify genetic mutations. In addition, the Human Gene Mutation Database (www.genecards.org) was used to determine whether each site was a novel mutation site.

\section{Results}

Clinical manifestion. All three of the patients with PPD were asymptomatic after birth with normal intellectual development. They possessed no distinctive facial features. The probands of families 1 and 3 were both the only male child of non-consanguineous healthy parents, and were delivered without complication at full-term. Their birth weight and length were within the normal limits. Stature was normal at onset of symptoms; however, a short trunk was observed at diagnosis in all three patients. The patient of family 2 was a fostered female child with unknown personal and family histories. In the patients from families 1 and 3 the initial symptoms were gait abnormalities and a degree of knee deformity. The initial exhibiting symptom in the patient of family 2 was swelling of the multiple interphalangeal joints. These three patients (two males and one female) were included in this study over a period of two years and nine months .These patients visited the clinic at the age of 11-21 years for short stature (current height, near or less than the standard deviation) and motion limitation of the joints. The patients were followed up for six to nineteen months. During this period, physical examinations and the routine blood tests (i.e., erythrocyte sedimentation rate, C-reactive protein, rheumatoid factor, serum calcium levels, serum phosphorus levels, alkaline phosphatase,parathyroid hormone, vitamin D status and insulin-like growth factor 1) were conducted. At the final follow-up appointment, all of the joints (small and large, including the spine) were progressively limited in movement. The family members of all three patients, including their parents, were not shown to exhibit any abnormal clinical, biochemical or radiographic manifestations. The detailed clinical data of the patients are presented in Table II.

Detection of mutations. Five WISP3 mutations were detected (two missense mutations, two nonsense mutations and one frameshift mutation). In family 1 , a compound heterozygous mutation was detected; the proband carried a novel missense mutation: c.667T $>\mathrm{G}$ (p.Cys223Gly) of the fourth exon in the maternal allele and a nonsense mutation: c. $857 \mathrm{C}>\mathrm{G}$ (p.Ser286*) of the fifth exon in the paternal allele (Fig. 2). A homozygous missense mutation: c.342T $>\mathrm{G}$ (p.Cys114Trp) was detected in the third exon of the patient from family 2 . A frameshift mutation and a nonsense mutation were detected in the fifth exon: c.866dupA (p.Ser290Glufs*13) and the second exon: c.136C $>\mathrm{T}$ (p.Gln46*) of the patient from family 3 , respectively. These two mutations formed a compound heterozygous mutation.

\section{Discussion}

The results of the present study are concordant with the findings of a previous study (5), with similar clinical manifestations. 
Table I. Primer sequences for the polymerase chain reaction amplification of the WISP3 gene.

\begin{tabular}{|c|c|c|c|c|c|}
\hline Amplicon & Primer sequence & $\mathrm{Tm}$ & $\begin{array}{c}\mathrm{GC} \\
\text { content }\end{array}$ & $\begin{array}{l}\text { Amplicon } \\
\text { size (bp) }\end{array}$ & $\begin{array}{l}\text { Annealing } \\
\text { temp. }\left({ }^{\circ} \mathrm{C}\right)\end{array}$ \\
\hline \multirow[t]{2}{*}{ WISP3-5'UTR\&EXON1 } & F-GTACGTGAGGGTGAAGCTGGA & 59.9 & 57.1 & & \\
\hline & R-GGAGAGACACTGTTTCCCGCA & 62.3 & 87.1 & 850 & 58 \\
\hline \multirow[t]{2}{*}{ WISP3-EXON2 } & F-TAAAGGAGGAGTAAGAGTGGA & 51.2 & 42.9 & & \\
\hline & R-CGGATTGTTTTTGACAGTATT & 52.5 & 33.3 & 868 & 50 \\
\hline \multirow[t]{2}{*}{ WISP3-EXON3 } & F-GGTGATTTTACAGGGTCTTTAC & 53 & 40.9 & & \\
\hline & R-ATTTATCCCTGTCTGAGGC & 51.2 & 47.4 & 667 & 50 \\
\hline \multirow[t]{2}{*}{ WISP3-EXON4 } & F-GAAAGAGGGAGATAGAGTGATA & 50 & 40.9 & & \\
\hline & R-GAAGTTAGAATCTGCTCTGGTT & 52.8 & 40.9 & 569 & 50 \\
\hline \multirow[t]{2}{*}{ WISP3-EXON5\&3'UTR } & F-GGTAAAGAGAGTGCTGGAAATC & 55.4 & 45.5 & & \\
\hline & R-TGCTTAGATGGTAACAAATGTC & 52.7 & 36.4 & 683 & 52 \\
\hline
\end{tabular}

UTR, untranslated region; F, forward; R, reverse; Tm, melting temperature; bp, base pairs; temp., temperature.

Table II. Basic clinical data of three patients with progressive pseudorheumatoid dysplasia.

\begin{tabular}{|c|c|c|c|}
\hline \multirow[b]{2}{*}{ Characteristic } & \multicolumn{3}{|c|}{ Patient } \\
\hline & 1 & 2 & 3 \\
\hline Gender & Male & Female & Male \\
\hline Family history & - & Unknown & - \\
\hline Age (years) & 13 & 11 & 21 \\
\hline Age at onset (years) & 6 & 4 & 3 \\
\hline Site of pathological changes & Knee joint & Interphalangeal joint & Knee joint \\
\hline Height $(\mathrm{cm})$ & $153.7(-1.2 \mathrm{SDS})$ & $135.3(-2.2 \mathrm{SDS})$ & $155-160(<2 S D S)$ \\
\hline Arthralgia & - & + & + \\
\hline Spinal nerve involvement & - & - & + \\
\hline Enlargement and stiffness of joint & + & ++ & +++ \\
\hline Activity limitation & Hip joint & Hip and knee joints & All joints \\
\hline Spine malformation & Lumbar lordosis & Scoliosis & Scoliosis \\
\hline Muscle atrophy & Lower limbs & Lower limbs & - \\
\hline Narrowing of intervertebral and joint space & + & ++ & +++ \\
\hline Vertebral deformities & + & ++ & +++ \\
\hline Acetabular fossa deformation & + & ++ & +++ \\
\hline Plain X-ray film of the skull & - & - & - \\
\hline Shortening of column femoris & + & ++ & +++ \\
\hline
\end{tabular}

SDS, height standard deviation score; 1, 2 and 3 indicate patients from families 1,2 and 3 respectively. '+': positive, '-': normal. Every additional ' + ' indicates that the disease condition gradually worsened at each follow up.

The clinical characteristics of PPD have been summarized in previous studies (4-8) and have been shown to worsen over time. As compared with the majority of skeletal dysplasias, prenatal skeletal growth and morphogenesis are not altered in PPD (2), and affected individuals are asymptomatic after birth, and usually suffer from the disease between the ages of 3 and 8 years old (4). The intellectual development of patients with PPD is normal. A normal life expectancy is estimated; however, joint contractures, fractures and spinal stenosis are major prognostic factors associated with PPD $(3,4,7)$. Gait abnormality is often observed in the initial period, and is followed by non-inflamma- tory and symmetrical joint enlargement, accompanied by pain, ankylosis, and limitation of activity, which may result in muscle atrophy, and finally disability $(3,4)$. Spine involvement develops after the age of 15 and adult height is usually below the 3rd percentile. Progressive, non-inflammatory multi-joint enlargement predominantly manifests as platyspondyly, epiphyseal (metaphysis) expansion and joint stenosis in medical imaging, without abnormalities detected by laboratory examination. $\mathrm{X}$-ray appearance of the head is normal $(3,4)$.

Due to the similar clinical symptoms and radiographic features, PPD is often clinically misdiagnosed, and should 
Table III. Key similarities and differences between PPD, SEDT and JIA.

PPD

Similarities

Short trunk, joint pain and activity

limitation, gait abnormality, similar vertebral

manifestation in X-ray film
JIA

SEDT
Arthralgia, swelling, activity limitation, growth delay

Differences

Femoral epiphysis becomes smaller instead of larger, which is accompanied by the enlargement of the little toe joint.

There is no spondyloepiphyseal dysplasia. Joint involvement may be asymmetric and accompanied by iridocyclitis, laboratory examination abnormalities. Anti-inflammatory and immunosuppressive therapies are effective.

PPD, progressive pseudorheumatoid dysplasia; SEDt, spondyloepophyseal dysplasia tarda; JIA, juvenile idiopathic arthritis. Abnormalities indicated by laboratory examination include: Anemia, increased peripheral leukocytes and platelets, enlargement of the lymph nodes, and increased erythrocyte sedimentation rate, C-reactive protein, rheumatoid factor and antinuclear antibodies $(4,21)$.

be distinguished from spondyloepiphyseal dysplasia tarda and juvenile idiopathic arthritis in the early stages of the disease (Table III) (9).

PPD is a rare genetic skeletal or cartilaginous dysplasia characterized by continuous loss of cartilage and bone destruction (10), due to the functional loss or abnormality of the WISP3 protein. PDD was initially reported in 1982 (1). In 1998, WISP3 was first cloned (11) and localized to the 6q22-6q23 chromosomal region. In 1999, using positional candidate cloning for the first time, Hurvitz et al (2) demonstrated that PPD was caused by WISP3 mutations. However, as compared with humans, WISP3-null mice and mice overexpressing WISP3 exhibit no gross abnormal phenotypes (12). WISP3 is an extracellular matrix (ECM)-associated protein; it is a small secreted cysteine-rich protein that is a member of the CCN protein family. WISP3 contains five exons and four introns, which encode 354 amino acids. CCN proteins are secreted cysteine-rich heparin binding glycoproteins that associate with the cell surface and extracellular matrix (13). WISP3 is composed of an N-terminal signal peptide; followed by four conserved cysteine-rich modular domains (which is known to be the unifying feature of the $\mathrm{CCN}$ protein family); an insulin-like growth factor binding protein domain (IGFBP) encoded by exon 2; a von Willebrand type $\mathrm{C}$ repeat (VWC) encoded by exon 3 , in which WISP3, unlike all of the other identified CCN family proteins, lacks 4 of 10 conserved cysteine residues (14); a thrombospondin type I domain (TSP-1) encoded by exon 4; and a cysteine knot carboxyl terminal (CT) encoded by exon 5. WISP3 triggers signal transduction in cell adhesion, migration, proliferation, differentiation and survival, through direct binding to specific integrin receptors and heparan sulfate proteoglycans (15). CCN proteins simultaneously integrate and modulate the signals of integrins, bone morphogenetic protein (BMP), vascular endothelial growth factor, Wnt and Notch by direct binding. The WISP3 gene usually inhibits cell growth (14); however, the role of WISP3 in the pathogenesis of PPD remains to be elucidated. Nakamura et al (16) revealed that WISP3 is involved in zebrafish cartilage development, and suggested that dysregulation of BMP and/or Wnt signaling may contribute to cartilage degeneration in humans with PPD (16). WISP3 is able to maintain cartilage stability by regulating the synthesis of type II collagen and aggrecan, two major matrix components of articular cartilage, in chondrocytes (17). Furthermore, it has been demonstrated that collagen II expression is downregulated in chondrocytes possessing a WISP3 mutation. Mutated WISP3 leads to decreased intracellular collagen II content, and secretion of extracellular collagen II with a delayed secretion peak, which is an important mechanism underlying the reduction of collagen size and density in the cartilage of patients with PPD (17,18). In addition, WISP3 mutations may result in continuous degeneration and loss of articular cartilage, which only affects the growth and differentiation of postnatal chondrocytes, without affecting fetal cartilage growth (2). This may partly explain the pathogenesis of PPD. Recent studies $(18,19)$ have shown that WISP3 may have a regulatory role through inhibiting the proliferation of chondrocytes, and promoting the differentiation of chondrogenic progenitor cells. Mutated WISP3 protein increased proliferative activity and decreased the rate of apoptosis of C-20/A4 cells (18), as well as aggregating abnormally in the cytoplasm. Therefore, it may be suggested that WISP3 mutations cause functional disorders, such as inhibition of chondrocyte proliferation and dysregulation of type II collagen expression, which may be involved in the pathogenesis of PPD (18).

Currently, 56 different WISP3 mutations have been reported globally, which are distributed in all coding exons of the WISP3 gene $(4-8,20)$. The mutations localized in exon 2 (15 types) and exon 5 (15 types) are predominant, followed by those in exon 4 (11 types). The mutation in exon 1 ( 1 type) is the least frequent. Another five types of mutations are localized in introns 1,2 and 3. The most common mutations are missense $(23 / 56,41 \%)$ and frameshift mutations $(20 / 56,36 \%)$. P.Cys52* may be considered the hotspot mutation, and is detected in various ethnic populations in the Middle East and Europe with the highest frequency $(4,5,6)$. However, this particular mutation has not been detected in China $(7,8,20)$. 

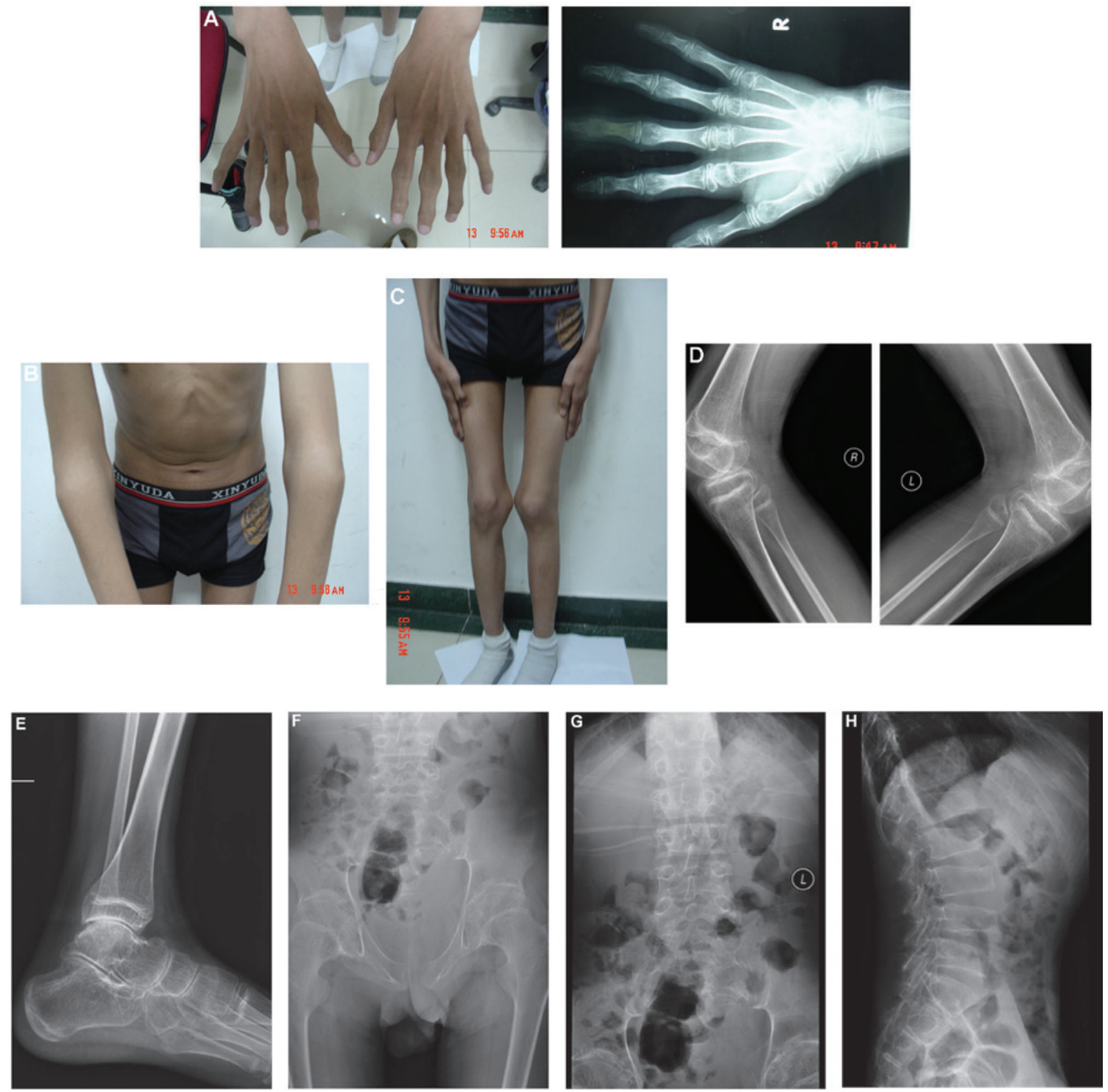

Figure 1. Images and X-rays of the proband from family 1. (A) Enlargement of the interphalangeal joints; hand radiograph demonstrates the enlargement of the metaphyses and epiphysis at the metacarpals and phalanges, with loss of joint space. (B) Enlargement of the bilateral elbow joints. (C and D) Slight enlargement of the knee epiphyses and metaphyses, and narrowing of the joint space with periarticular osteoporosis. (E) Flattened talus with narrow joint spaces. (F) Deepening of the acetabular bone, narrowing of the hip joint space, enlargement and roughness of the femoral head, and shortening of the femoral neck. (G) Narrowing of the intervertebral space, platyspondyly. (H) Flattened vertebrae on the thoracic and lumbar spine with anterior beaking and an increased anteroposterior diameter of the vertebral body.

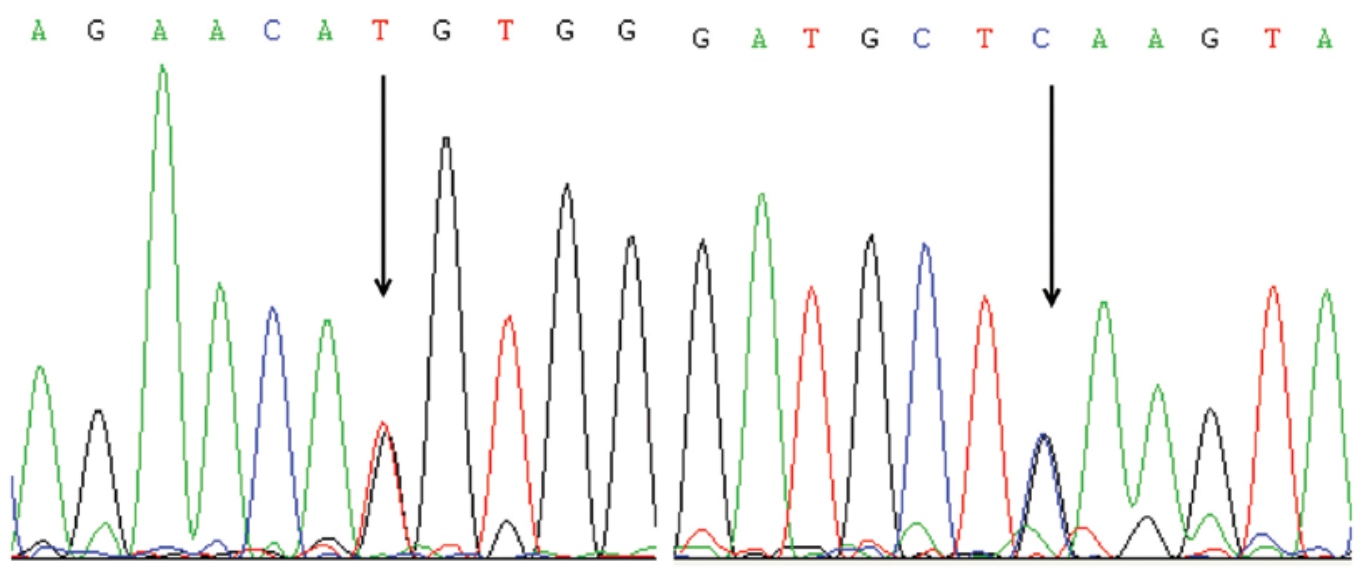

Figure 2. Automated sequencing traces of the WISP3 gene mutations in the proband of family 1. Arrows indicate mutations. 
In the present study, two missense mutations, two nonsense mutations and one frameshift mutation were detected. Compound heterozygosity (c.667T $>$ G/c.857C $>$ G) of WISP3 was identified in family 1 , where the proband possessed a novel missense mutation c.667T $>$ G (p.Cys223Gly) in the fourth exon of the maternal allele, and a nonsense mutation c. $857 \mathrm{C}>\mathrm{G}$ (p.Ser286*) in the fifth exon of the paternal allele. This sequence variation was not present in any of the control samples, which indicates that this variation is not a single nucleotide polymorphism. As a novel missense mutation c.667T>G, $\mathrm{T}$ at position 667 was replaced by $\mathrm{G}$ due to this mutation. This resulted in codon 223 changing from TGT (coding Cys) to GGT (coding Gly), which led to PPD in a compound heterozygous mutation state. The WISP3 protein also has a series of primary structures consisting of 34 cysteines, which is similar to other proteins in the CCN family (14). These primary structures are conserved in number and position, as part of the sequence motif forming each structural domain of WISP3. A change in any cysteine residue can seriously interfere with the biological functions of the protein; for example, regulation of BMP and/or Wnt signaling, resulting in PPD when present in the homozygote or compound heterozygote state (16). The unique feature of these $\mathrm{CCN}$ family proteins is a multidomain structure with an N-terminal secretory signal peptide and four functional domains (IGFBP, VWC, TSP-1 and CT) (14). This novel missense mutation is located in the TSP-1 domain, which binds heparin or sulfated proteoglycans in order to modulate cell adhesion and maintain ECM composition. The nonsense mutation c. $857 \mathrm{C}>\mathrm{G}$ (p.Ser286*), produces a truncated WISP3 protein (p.Ser286*) composed of 285 amino acid residues, was detected for the first time in Chinese patients in the present study. The nonsense mutation c. $857 \mathrm{C}>\mathrm{G}$ has only previously been reported to be homozygous in an affected individual in Turkey, and patients carrying a missense mutation in one of the two alleles did not show any significant difference in severity of the PPD phenotype, as compared with patients possessing two nonsense mutations in both alleles (4). The phenotype of the male from family 1 in this study was no milder than that of the Turkish patient. This nonsense mutation belongs to the fourth (CT) domain, which is involved in disulfide-linked dimerization and is required for dimer formation in the endoplasmic reticulum, an important function for the establishment and maintenance of the normal 3D-conformation of WISP3 protein (18). CT domain-mediated dimerization has been suggested to act in synergy with TSP-domain-mediated oligomerization, in order to give rise to the large $\mathrm{CCN}$ oligomers, and may be an important factor in directing how $\mathrm{CCN}$ proteins control and manipulate adhesion processes and ECM composition, and mediate cell adhesion (1). Therefore, the present study hypothesized that the compound heterozygous mutation formed a truncated WISP3 protein and Cys223Gly mutated protein, and that the two mutated WISP3 proteins possibly aggregate abnormally in the cytoplasm, inducing morphological transformation of chondrocytes. It has been reported worldwide in exons 4 and 5, that the majority of mutations are either frameshift or missense mutations (26/56, $46 \%$ ), or those involving cysteine residues (6). These findings indicate that the inhibitory effect of WISP3 on cell proliferation requires the expression of a full length protein and proper protein folding. The present study also detected the following three recurrent mutations: Homozygous missense mutation \{c.342T>G (p.Cys114Trp)\} of the patient from family 2, and a compound heterozygous mutation, the nonsense mutation c.136C >T (p.Gln46*) and the duplication mutation c.866dupA (p.Ser290Glufs*13) of the proband from family 3 . The pathogenesis described above requires confirmation through further studies regarding protein expression.

A total of 12 types of mutations (six frameshift mutations, five missense mutations and two nonsense mutations) have previously been detected in patients with PPD in China $(8,20)$. These include four different mutations (three frameshift mutations and one missense mutation) localized in exon 4 and in exon 5 (two missense mutations, one frameshift mutation and one nonsense mutation) respectively, as well as three mutations in exon 2 (two missense mutations and one nonsense mutation). However, there have been no WISP3 mutations reported in exon 3 in Chinese patients. Currently, the most common mutations are frameshift and missense $(10 / 12,83 \%)$. In addition, the mutation c.624_625dupA in exon 4, two mutations c.866dupA and c.1000T $>C$ in exon 5, and mutation c.136C $>\mathrm{T}$ in exon 2 are most commonly reported $(7,8,20)$. Therefore, it may be hypothesized that these four types of mutations may be hot spot mutations of WISP3 in Chinese populations.

There are currently no effective treatments for PPD, which is the reason for the high disability rate associated with the disease, despite its rarity. The disease can be diagnosed based on typical clinical manifestations, and X-ray and biochemical examination. A definitive diagnosis can be made at the molecular biology level through analysis of WISP3 mutations. In the present study, the three patients possessed similar clinical phenotypes, and no specific correlation was observed between genotype and phenotype. These results support the conclusion that various WISP3 mutations may result in similar clinical phenotypes (4).

In conclusion, a novel mutation c.667T $>\mathrm{G}$ (p.Cys223Gly) and the c.857C $>\mathrm{G}$ (p.Ser286*) mutation were detected in three Chinese patients with PPD, alongside three recurrent mutations. The clinical phenotypes of PPD were relatively consistent, and the patients exhibited the characteristic clinical and radiographic findings associated with PPD. Therefore, imaging examinations have diagnostic significance for PPD; however, the final diagnosis must rely on the detection of WISP3 mutations.

\section{Acknowledgements}

The authors would like to thank the patients and their family members for actively participating in the study. They are particularly grateful to the patients and their family members who provided images for the present study.

\section{References}

1. Wynne-Davies R, Hall C and Ansell BM: Spondylo-epiphysial dysplasia tarda with Progressive arthropathy. A "new" disorder of autosomal recessive inheritance. J Bone Joint Surg Br 64: 442-445, 1982

2. Hurvitz JR, Suwairi WM, Van Hul W, et al: Mutations in the CCN gene family member WISP3 cause progressive pseudorheumatoid dysplasia. Nat Genet 23: 94-98, 1999.

3. Bennani L, Amine B, Ichchou L, Lazrak N and Hajjaj-Hassouni N: Progressive pseudorheumatoid dysplasia: three cases in one family. Joint Bone Spine 74: 393-395, 2007. 
4. Garcia Segarra N, Mittaz L, Campos-Xavier AB, et al: The diagnostic challenge of progressive pseudorheumatoid dysplasia (PPRD): a review of clinical features, radiographic features, and WISP3 mutations in 63 affected individuals. Am J Med Genet C Semin Med Genet 160C: 217-229, 2012.

5. Delague V, Chouery E, Corbani S, et al: Molecular study of WISP3 in nine families originating from the Middle-East and presenting with progressive pseudorheumatoid dysplasia: identification of two novel mutations and description of a founder effect. Am J Med Genet A 138A: 118-126, 2005.

6. Dalal A, Bhavani G SL, Togarrati PP, et al: Analysis of the WISP3 gene in Indian families with progressive pseudorheumatoid dysplasia. Am J Med Genet A 158A: 2820-2828, 2012.

7. Sun J, Xia W, He S, et al: Novel and recurrent mutations of WISP 3 in two Chinese families with progressive pseudorheumatoid dysplasia. PLoS One 7: e38643, 2012.

8. Ye J, Zhang HW, Wang T, et al: Clinical diagnosis and WISP3 gene mutation analysis for progressive pseudorheumatoid dysplasia. Zhonghua Er Ke Za Zhi 48: 194-198, 2010 (In Chinese)

9. Shivanand G, Jain V and Lal H: Progressive pseudorheumatoid chondrodysplasia of childhood. Singapore Med J 48: e151-e153, 2007.

10. No authors listed: International nomenclature and classification of the osteohondrodysplasias (1997): International Working Group on Constitutional Diseases of Bone. Am J Med Genet 79 376-382, 1998.

11. Pennica D, Swanson TA, Welsh JW, et al: WISP3 genes are members of the connective tissue growth factor family that are up-regulated in wnt-1-transformed cells and aberrantly expressed in human colon tumors. Proc Natl Acad Sci USA 95: 14717-14722, 1998.

12. Kutz WE, Gong Y and Warman ML: WISP3, the gene responsible for the human skeletal disease progressive pseudorheumatoid dysplasia, is not essential for skeletal function in mice. Mol Cell Biol 25: 414-421, 2005.
13. Zuo GW, Kohls CD, He BC, et al: The $\mathrm{CCN}$ proteins: important signaling mediators in stem cell differentiation and tumorigenesis. Histol Histopathol 25: 795-806, 2010.

14. Holbourn KP, Acharya KR and Perbal B: The CCN family of proteins: structure-function relationships. Trends Biochem Sci 33: 461-473, 2008

15. Chen PC, Cheng HC, Yang SF, Lin CW and Tang CH: The CCN family proteins: modulators of bone development and novel targets in bone-associated tumors. Biomed Res Int 2014: 437096 , 2014.

16. Nakamura Y, Weidinger G, Liang JO, et al: The CCN family member Wisp3, mutant in progressive pseudorheumatoid dysplasia, modulates BMP and Wnt signaling. J Clin Invest 117: 3075-3086, 2007.

17. Sen M, Cheng YH, Goldring MB, Lotz MK and Carson DA: WISP3-dependent regulation of type II collagen and aggrecan production in chondrocytes. Arthritis Rheum 50: 488-497, 2004.

18. Wang M, Man XF, Liu YQ, et al: Dysfunction of collagen synthesis and secretion in chondrocytes induced by wisp3 mutation. Int J Endocrinol 2013: 679763, 2013.

19. Wang M, Peng YQ, Zhou HD, Zhai MX, He YL and Xie H: Construction of WISP3 gene's mutants in SEDT-PA and their expression in COS-7 cells. Zhong Nan Da Xue Xue Bao Yi Xue Ban 33: 8-15, 2008 (In Chinese).

20. Yue H, Zhang ZL and He JW: Identification of novel mutations in WISP3 gene in two unrelated Chinese families with progressive pseudorheumatoid dysplas. Bone 44: 547-554, 2009.

21. Ye J, Zhang HW, Qiu WJ, et al: Patients with progressive pseudorheumatoid dysplasia: from clinical diagnosis to molecular studies. Mol Med Rep 5: 190-195, 2012. 